\title{
AN OPERATIONAL CALCULUS FOR MEROMORPHIC FUNCTIONS
}

\author{
HERBERT A. GINDLER*
}

Consider a linear transformation $T$ whose domain $D(T)$ and range $R(T)$ lie in a complex Banach space $X$. We denote by $[X]$ the class of all linear transformations $T$ for which $D(T)=X$ and $T$ is continuous (bounded). Also, we denote the resolvent set of $T$ by $\rho(T)$ and the spectrum of $T$ by $\sigma(T)$.

In 1943 Nelson Dunford [1 and 2] and A. E. Taylor [1] developed a method of associating with each holomorphic function $f$ of a certain class an operator in $[X]$ denoted by $f(T)$ where $T \in[X]$. This mapping $f \rightarrow f(T)$ gives what is commonly called an operational calculus. Subsequently, A. E. Taylor [2] extended these results to include the case where $T$ is closed in the sense that the graph of $T$ is a closed subset of $X \times X$ and $\rho(T)$ is not empty. One fact of great importance is that the spectrum of $f(T)$ is determined by $f$ and $\sigma(T)$. (See Hille and Phillips [1, p. 204].) It is the purpose of this paper to extend these results to a certain class of meromorphic functions.

We shall now describe briefly the operational calculus as developed by Taylor. We assume that $X$ is a Banach space and that $T$ is a closed linear operator so that $R(\lambda ; T)=(\lambda-T)^{-1} \in[X]$ if $\lambda \in \rho(T)$, and $R(\lambda ; T)$ is analytic as a function from $\rho(T)$ to $[X]$.

Let $f$ be a complex-valued function which is holomorphic (locally analytic) on an open set $\Delta(f)$ which contains $\sigma(T) \cup\{\infty\}$. We require $\Delta(f)$ to be open in the extended plane, and $f$ must be regular at $\infty$. Given such an $f$, we define $f(T)$ as follows:

$$
f(T)=f(\infty) I+\frac{1}{2 \pi i} \int_{\partial \nu} f(\lambda) R(\lambda ; T) d \lambda,
$$

where $\partial D$ is the oriented boundary of a suitable domain $D$. By "suitable" we mean the following: $D$ is an unbounded Cauchy domain which contains $\sigma(T)$

Received September 22, 1964.

* The author wishes to acknowledge his indebtedness to A. E. Taylor for his help and encouragement. 
and is such that $\bar{D} \subset \Delta(f)$. A Cauchy domain is an open set in the plane, with a finite number of components, and it is such that $\partial D$ consists of a finite positive number of closed rectifiable curves, no two of which intersect. The orientation of $\partial D$ is the usual positive orientation of the boundary. With $D$ unbounded there will be just one unbounded component, and its complement will be compact. Thus, with the situation we have, if we add the point $\infty$ to $D$ and look at $D$ as a set in the extended plane, then $D$ is a neighborhood of $\sigma(T) \cup\{\infty\}$.

Under these conditions $f(T)$ is a member of $[X]$. It is independent of the particular choice of $D$.

Two functions $f, g$, each of the general character here considered, may be considered equivalent if they agree on a neighborhood of $\sigma(T) \cup\{\infty\}$. In that case $f(T)=g(T)$. We can form equivalence classes of functions, and these equivalence classes form a ring in a natural way. We denote the ring by $\mathscr{A}(T)$. If $f$ is a representative of an equivalence class, the mapping $f \rightarrow f(T)$ turns out to yield a ring homomorphism of $\mathscr{A}(T)$ into $[X]$. Therein lies the essence of the operational calculus.

In order to state precisely the relationship between $\sigma(T)$ and $\sigma(f(T))$ the properties $P_{i}$ as in the following definition will be used.

Definition 1. The properties $P_{i}(i=1,2,3)$ for the operator $T$ are defined as follows, on the assumption that $T$ is linear, with domain and range in $X$.

$P_{1}: T$ is not one-to-one.

$P_{2}$ : The range of $T$ is not dense in $X$.

$P_{3}$ : There exists a sequence of unit vectors $x_{n}$ for which $\left\|T x_{n}\right\| \rightarrow 0$ as $n \rightarrow \infty$.

It is not difficult to verify that $\lambda \in \sigma(T)$ if and only if $\lambda-T$ has the property $P_{i}$ for some $i=1,2$ or 3 .

Theorem 1. (The fine-point spectral mapping theorem.) Let $X$ be a Banach space, let $T$ be a closed linear operator with a nonempty resolvent set, and suppose $f \in \mathscr{H}(T)$. If $\alpha-T$ has the property $P_{i}(i=1,2$ or 3$)$ then so has $f(\alpha)-f(T)$. If $\mu-f(T)$ has the property $P_{i}(i=1,2$ or 3$)$, if $\mu \neq f(\infty)$ and if $f(\lambda) \neq \mu$ on each component of the domain of $f$, then there exists an $\alpha$ in $\sigma(T)$ such that $f(\alpha)$ $=\mu$ and $\alpha-T$ has the property $P_{i}$. If $T \in[X]$, then $\mu=f(\infty)$ need not be 
excepted.

This theorem is proved in Hille and Phillips (pp. 204 and 205). The purpose of this paper is to prove Theorem 1 for a more comprehensive class of functions which we will now describe.

Definition 2. We shall refer to the body of theory developed in the rest of this paper as the "operational calculus for meromorphic functions".

Suppose $X$ is a Banach space and let $T$ be a closed linear operator with a non-empty resolvent set. Let $f$ be a meromorphic function whose domain is an open set containing $\sigma_{e}(T)=\sigma(T) \cup\{\infty\}$. Since $\sigma_{e}(T)$ is a compact subset of the extended plane, $f$ will have (at most) a finite number of poles, $\alpha_{1}, \alpha_{2}$, $\ldots, \alpha_{k}$, on $\sigma(T)$. Let $n_{i}$ be the order of the pole $\alpha_{i}$ for $i=1,2, \ldots, k$ and let $n_{0}$ be the order of $\infty$ as a pole of $f$. We further require that $\left(\alpha_{i}-T\right)^{-1}$ exists at each finite pole of $f$. Define the polynomial $p$ by

$$
p(\lambda)=\left(\alpha_{1}-\lambda\right)^{n_{1}} \cdots\left(\alpha_{k}-\lambda\right)^{n_{k}}
$$

let $m=n_{1}+\cdots+n_{k}$ be the degree of $p$, let $n=n_{0}+m$ and suppose $\beta \in \rho(T)$. The function $F$ given by

$$
\begin{aligned}
F(\lambda)=f(\lambda) p(\lambda)(\beta-\lambda)^{-n} \text { for } \lambda \neq \alpha_{i}, F\left(\alpha_{i}\right) & =\lim _{\lambda \rightarrow \alpha_{i}} F(\lambda) \\
& \text { for } i=1, \ldots, k
\end{aligned}
$$

is holomorphic (locally analytic) on an open set containing $\sigma_{e}(T)$. We can now define $F(T)$ in $[X]$ by use of the operational calculus for holomorphic functions in $\mathscr{H}(T)$. Also $p(T)[R(\beta ; T)]^{n}$ has an inverse which will be denoted by $S(\beta ; T)$ to emphasize its dependence on $\beta$ and $T$ as well as to preserve the analogy with the notation $R(\beta ; T)$ for $(\beta-T)^{-1}$. We denote by $\mathscr{M}(T)$ the class of meromorphic functions satisfying all the requirements listed in this paragraph. Finally, for each $f \in \mathscr{M}(T)$ we define the linear operator $f(T)$ by

$$
f(T) x=F(T) S(\beta ; T) x \quad \text { for } x \text { in the domain of } S(\beta ; T) .
$$

Observe that, if $f \in: \mathscr{K}(T)$ but $f$ has no poles on $\sigma_{e}(T)$, equation (3) defines the same operator $f(T)$ as that guaranteed by the operational calculus for holomorphic functions in $\mathscr{H}(T)$. But, unlike this earlier operational calculus, equation (3) does not define an element of $[X]$, in general. In fact, $f(T) \in$ 
$[X]$ if and only if $f$ is locally analytic on an open set containing $\sigma_{e}(T)$. This does not deny the possibility that $f(T)$ be bounded on its domain as will be the case whenever $\infty$ is not a pole of $f$ and $\alpha_{i}-T$ has a bounded inverse for each finite pole of $f$.

We proceed now with the task of developing the machinery necessary to prove a fine-point spectral mapping theorem for members of $\mathscr{M}(T)$. We employ the notation introduced earlier.

Lemma 2. Let $X$ be a Banach space and let $T$ be a closed linear operator from $X$ to $X$. If $\left\{y_{k}\right\}$ is a sequence of unit vectors in the domain of $T$ for which $(\alpha-T) y_{k} \rightarrow 0$ as $k \rightarrow \infty$, then, for each positive integer $n$ and each $\beta$ in $\rho(T)$, $\left\|[R(\beta ; T)]^{n-1} y_{k}\right\|$ is bounded away from zero.

Proof. The proof is by induction. The case $n=1$ is trivial, so we may assume $n>1$ and that $\left\|[R(\beta ; T)]^{n-2} y_{k}\right\|$ is bounded away from zero. Then, writing $x_{k}=[R(\beta ; T)]^{n-1} y_{k}$, we have

$$
\begin{aligned}
\left\|(\beta-\alpha) x_{k}\right\| \geq\left\|(\beta-T) x_{k}\right\|-\left\|(\alpha-T) x_{k}\right\|= \\
\quad=\left\|[R(\beta ; T)]^{n-2} y_{k}\right\|-\|\left[R(\beta ; T)^{n-1}(\alpha-T) y_{k} \| .\right.
\end{aligned}
$$

The second term on the right hand side of this equation goes to zero, while the first term on the right is bounded away from zero. It follows that $\left\|x_{k}\right\|$ is bounded away from zero, as was to be proved.

Lemma 3. Let $X$ be a Banach space, let $T$ be a closed linear operator with a non-empty resolvent set, and suppose $f \in \mathscr{M}(T)$. Then, for $f(T), \beta, S(\beta ; T)$ and $F(T)$ as in equation (3),

$$
f(T) x=S(\beta ; T) F(T) x \text { for all } x \text { in the domain of } f(T)
$$

Hence $f(T)$ is closed in the sense that the graph of $f(T)$ is closed in $X \times X$.

Proof. The operator $S(\beta ; T)$ is closed, for its inverse $p(T)[R(\beta ; T)]^{n}$ is in $[X]$. Since $F(T) \in[X]$, it will follow that $f(T)$ is closed once equation (4) is established.

We know that

$$
p(T)[R(\beta ; T)]^{n} F(T) x=F(T) p(T)[R(\beta ; T)]^{n} x
$$

for all $x$ in $X$. Letting $y=p(T)[R(\beta ; T)]^{n} x$ or $x=S(\beta ; T) y$ in equation (5) and applying $S(\beta ; T)$ to both sides of this equation, we obtain 
$F(T) S(\beta ; T) y=S^{\prime}(\beta ; T) F(T) y \quad$ for all $y$ in the domain of $S(\beta ; T)$.

In view of the definition of $f(T)$, this establishes equation (4).

The following lemma will also prove useful.

Lemma 4. Let $f$ and $g$ be in $\mathscr{M}(T)$, with $X$ and $T$ as in the previous lemma, and suppose $g$ has the same poles with the same order as $f$. Then $h=f+g \in \mathscr{M}(T)$ and, for each $\beta$ in $\mu(T), h(T)$ is an extension of $f(T)+g(T)$. If $g \in \mathscr{A}(T)$, then $h(T)=f(T)+g(T)$.

Proof. Let $q$ be a polynomial related to the poles of $h$ in the same way that the polynomial $p$ in the discussion preceding equation $(3)$ is related to the poles of $f$. It is not necessarily true that $q=p$ but $q$ does divide $p$ so that, since $q \neq 0, r=p / q$ is a polynomial. We now define the holomorphic functions $F, G$ and $H$ by

$$
\begin{aligned}
& F(\lambda)=f(\lambda) p(\lambda)(\beta-\lambda)^{-n} \\
& G(\lambda)=g(\lambda) p(\lambda)(\beta-\lambda)^{-n} \\
& H(\lambda)=h(\lambda) q(\lambda)(\beta-\lambda)^{-k}
\end{aligned}
$$

where each of the functions $F, G$ and $H$ are defined by the appropriate limits at the poles of $f$ and where $n$ and $k$ are related to $f$ and $h$ in the same way $n$ is related to $f$ in the discussion leading up to equation (3). Clearly, $F, G$ and $H$ are members of $\mathscr{H}(T)$ and

$$
H(\lambda) r(\lambda)(\beta-\lambda)^{k-n}=F(\lambda)+G(\lambda) .
$$

By expressing $r(\lambda)(\beta-\lambda)^{k-n}$ as a polynomial in $(\beta-\lambda)^{-1}$, applying the operational calculus and then factoring out $[R(\beta ; T)]^{n-k}$, we obtain $r(T)[R(\beta ; T)]^{n-k}$ as a member of the operational calculus. It now follows that

$$
H(T) r(T)[R(\beta ; T)]^{n-k}=F(T)+G(T) .
$$

For $x$ in the domain of $f(T)$, write

$$
x=p(T)[R(\beta ; T)]^{n} y
$$

or

$$
y=S(\beta ; T) x
$$

Using first the definition of $f(T)$ and $g(T)$ and equation (7), then equation (6), then the relationship between $H(T)$ and $h(T)$, and finally the relationship 
between $r, q$ and $p$ and equation (8), we have

$$
\begin{aligned}
{[f(T)+g(T)] \dot{x} } & =[F(T)+G(T)] y=H(T) r(T)[R(\beta ; T)]^{n-k} y \\
& =h(T) q(T)[R(\beta ; T)]^{k} r(T)[R(\beta ; T)]^{n-k} y \\
& =h(T) x \text { for all } x \text { in the domain of } f(T)+g(T) .
\end{aligned}
$$

This shows that $h(T)$ is an extension of $f(T)+g(T)$. If $g \in \mathscr{A}(T)$, the same calculations can be performed but in this case $q=p$ and $n=k$ so that $h(T)$ has the same domain as $f(T)$.

We are now ready to prove the promised extension of the fine-point spectral mapping theorem. Theorem 5 relies very heavily on Theorem 1 as well as the notation and lemmas following Definition 2.

TheOREM 5. Let $X$ be a Banach space. Let $T$ be a closed linear operator with a non-empty resolvent set and suppose $f \in \mathscr{M}(T)$. If $\alpha \in \sigma(T)$ but $\alpha$ is not a pole of $f$ and if $\alpha-T$ has the property $P_{i}(i=1,2$ or 3$)$, then so has $f(\alpha)-f(T)$, where $f(T)$ is defined by equation (3). If $\mu-f(T)$ has the property $P_{i}$, if $\mu \neq f(\infty)$ and if $f(\lambda) \neq \mu$ on each component of the domain of $f$, then there exists an $\alpha$ in $\sigma(T)$ such that $f(\alpha)=\mu$ and $\alpha-T$ has the property $P_{i} . \quad$ If $T \in[X]$, then $\mu=f(\infty)$ need not be excepted.

Proof. If $\alpha \in \sigma(T)$ and $\alpha$ is not a pole of $f$,

$$
G(\lambda)=[f(\alpha)-f(\lambda)] p(\lambda)(\beta-\lambda)^{-n} \in \mathscr{\&}(T) .
$$

Now $h(\lambda)=f(\alpha)-f(\lambda)$ satisfies the hypotheses of the previous lemma with $f(\alpha)$ constant, hence analytic, so that $h(T)=f(\alpha)-f(T)$ by Lemma 4. On the other hand, $h \in \mathscr{M}(T)$ and $h(T)=G(T) S(\beta ; T)$ by definition. Therefore,

$$
[f(\alpha)-f(T)] x=G(T) S(\beta ; T) x
$$

for all $x$ in the domain of $f(T)$.

The remainder of this part of the proof rests heavily on the fact that the function $G$ is known to satisfy the fine-point spectral mapping theorem (Theorem 1) and the fact that $G(\alpha)=0$.

Suppose $\alpha-T$ has the property $P_{1}$, so that there exists a $y \in X$ such that $G(T) y=0$ but $y \neq 0$. Letting $x=p(T)[R(\beta ; T)]^{\prime} y$, we see that $[f(\alpha)-f(T)] x$ $=0$ by equation (9). We know $x \neq 0$ because of the restrictions placed on $p$ earlier. This shows that the assertion of the first part of the theorem holds for the property $P_{1}$. 
From equation (9) we see that the range of $f(\alpha)-f(T)$ is contained in the range of $G(T)$, so that $f(\alpha)-f(T)$ does not have a dense range if the range of $G(T)$ is not dense. The same relationship holds between $G(T)$ and $\alpha-T$. Hence, $f(\alpha)-f(T)$ has the property $P_{2}$ if $\alpha-T$ has this property.

Suppose there exist unit vectors $y_{k}, k=1,2, \ldots$, with each $y_{k}$ in the domain of $T$ and such that $(\alpha-T) y_{k} \rightarrow 0$ as $k \rightarrow \infty$. Then $G(T) y_{k} \rightarrow 0$. Let

$$
x_{k}=p(T)[R(\beta ; T)]^{n} y_{k} \quad \text { for } k=1,2, \ldots
$$

Then $x_{k}$ is in the domain of $f(T)$ for each $k$ and $[f(\alpha)-f(T)] x_{k}=G(T) y_{k} \rightarrow 0$ as $k \rightarrow \infty$. The desired conclusion will follow once it is shown that the $\left\|x_{k}\right\|$ are bounded away from zero. To see that this is the case, write

$$
p(T)=q(T)(\alpha-T)+\delta
$$

where $q$ is a suitable polynomial and $\delta$ is a complex number. Observe that $\delta \neq 0$ because $\alpha$ is not a pole of $f$ by hypothesis, so $\alpha-\lambda$ is not a factor of $p(\lambda)$. Using the above expression for $p(T)$, the definition of $x_{k}$ and applying the triangular inequality we have

$$
\begin{aligned}
\left\|x_{k}\right\|_{1} & =\left\|p(T)[R(\beta ; T)]^{n} y_{k}\right\| \\
& \geq|\delta|\left\|[R(\beta ; T)]^{n} y_{k}\right\|-\left\|q(T)[R(\beta ; T)]^{n}(\alpha-T) y_{k}\right\| .
\end{aligned}
$$

The $\left\|[R(\beta ; T)]^{n} y_{k}\right\|$ are bounded away from zero by Lemma 2 . Also,

$$
\left\|q(T)[R(\beta ; T)]^{n}(\alpha-T) y_{k}\right\|_{1} \rightarrow 0 \quad \text { as } k \rightarrow \infty,
$$

because $q(T)[R(\beta ; T)]^{n} \in[X]$ and $(\alpha-T) y_{k} \rightarrow 0$ as $k \rightarrow \infty$. Since $\delta \neq 0$, we conclude from (7) that the $\left\|x_{k}\right\|$ are bounded away from zero. Hence $f(\alpha)-f(T)$ has the property $P_{3}$.

To show the converse, suppose $\mu \equiv \sigma(f(T))$ and suppose $f(\lambda) \neq \mu$ on any component of $\sigma(T)$. Let

$$
G(\lambda)=[\mu-f(\lambda)] p(\lambda)(\beta-\lambda)^{-n} .
$$

Again the proof relies heavily on the fact that $G$ satisfies the fine-point spectral mapping theorem. If $\mu-f(T)$ has the property $P_{i}(i=1,2$ or 3$)$, we shall show that $G(T)$ also has the property $P_{i}$. It will then follow that there exists a complex number $\alpha$ such that $G(\alpha)=0$ and $\alpha-T$ has the property $P_{i}$. Since $G(\alpha)=0$ implies $\mu=f(\alpha)$, all will be proved.

We now propose to present the proof promised in the preceding paragraph. 
Suppose $x$ is in the domain of $f(T), x \neq 0$ and $[\mu-f(T)] x=0$. By Lemma 4,

$$
G(T) S(\beta ; T) x=[\mu-f(T)] x
$$

so that $G(T)$ annihilates the non-zero vector $S(\beta ; T) x$. This shows that $G(T)$ has the property $P_{1}$ if $\mu-f(T)$ does.

If $y \in X$, then $x=p(T)[R(\beta ; T)]^{n} y$ is in the domain of $f(T)$ and

$$
G(T) y=[\mu-f(T)] x .
$$

This shows the range of $G(T)$ is contained in the range of $\mu-f(T)$. The desired conclusion about the property $P_{2}$ now follows.

Similarly, let $\left\{x_{k}\right\}$ be a sequence of unit vectors contained in the domain of $f(T)$, and suppose $[\mu-f(T)] x_{k} \rightarrow 0$ as $k \rightarrow \infty$. There exist $y_{k}$ for which

$$
x_{k}=p(T)[R(\beta ; T)]^{n} y_{k} .
$$

Then

$$
G(T) y_{k}=G(T) S(\beta ; T) x_{k}=[\mu-f(T)] x_{k},
$$

so that $G(T) y_{k} \rightarrow 0$ as $k \rightarrow \infty$. Moreover, by expressing $p(T)[R(\beta ; T)]^{n}$ as a polynomial in $R(\beta ; T)$ we see that $p(T)[R(\beta ; T)]^{n} \in[X]$, and

$$
1=\left\|x_{k}\right\| \leq\left\|p(T)[R(\beta ; T)]^{n}\right\|\left\|y_{k}\right\| .
$$

This shows that the $\left\|y_{k}\right\|$ are bounded away from zero. Hence, if $\mu-f(T)$ has the property $P_{3}$, then so does $G(T)$.

The proof of the theorem can now be completed as indicated above.

\section{BIBLIOGRAPHY}

[1] Nelson Dunford,

1. Spectral theory. I. Convergence to projections. Trans. Amer. Math. Soc., vol. 54 (1943), pp. 185-217.

2. Spectral theory. Bull. Amer. Math. Soc., vol. 49 (1943), pp. 637-651.

[2] E. Hille and R. S. Phillips,

1. Functional Analysis and Semi-Groups, American Math. Soc. Colloquium Publications, vol. XXXI, Revised Ed. 1957.

[3] A. E. Taylor,

1. Analysis in complex Banach spaces. Bull. Amer. Math. Soc., vol. 49 (1943), pp. 652-669.

2. Spectral theory of closed distributive operators, Acta. Math., vol. 84 (1950), pp. 189224.

San Diego State College and University of Pittsburgh 